\title{
GAAS DISPLACEMENT DAMAGE DOSIMETER BASED ON DIODE DARK CURRENTS
}

\author{
Jeffrey H. Warner ${ }^{1}$, Scott R. Messenger ${ }^{2}$, Cory D. Cress ${ }^{1}$, Nicolas J-H Roche ${ }^{3}$, Michael Yakes ${ }^{1}$, Kenneth Clarke ${ }^{1}$, \\ Mitchell F. Bennett ${ }^{4}$, Phillip P. Jenkins ${ }^{1}$, Robert J. Walters ${ }^{1}$ \\ ${ }^{(1)}$ US Naval Research Laboratory,Washington,DC 20375 (USA),Email: dd-effects@nrl.navy.mil \\ ${ }^{(2)}$ Northrup Grumman, Baltimore, MD 21240 \\ (3) The George Washington University, Washington, DC 20375 \\ (4) Sotera Defense Solutions, Annapolis Junction, MD 20701
}

\begin{abstract}
GaAs diode dark currents are correlated over a very large proton energy range as a function of displacement damage dose (DDD). The linearity of the dark current increase with DDD over a wide range of applied voltage bias deems this device an excellent candidate for a displacement damage dosimeter. Additional proton testing performed in situ enabled error estimate determination to within $10 \%$ for simulated space use.
\end{abstract}

\section{INTRODUCTION}

The concepts of nonionizing energy loss (NIEL) and displacement damage dose (DDD) have been shown to be useful for correlating displacement damage produced by different particles in irradiated semiconductor [1-9]. This research has culminated over the past few years in the context of solar cell degradation in the production of a MATLAB-based software package called Solar Cell Radiation Environment Analysis Models (SCREAM) [10, 11]. SCREAM has been very successful in providing valid explanations involving several high profile space programs such as GPS [12] and TacSat4 [13]. In GPS spacecraft, there has been an anomalous degradation of the solar arrays since the beginning of the program, not agreeing with those results as predicted using the existing environment models [4]. SCREAM was used to calculate the expected damage on shielded silicon arrays using on-orbit dosimetry provided by BDD detectors from Los Alamos National Laboratories. The BDD data were used as input to provide a time dependent accumulation of the DDD behind the coverglass and adhesive shielding and the associated solar cell degradation was then determined. In this case the onorbit solar cell degradation and model calculations remained to disagree which effectively eliminated the cell degradation as the issue. For the TacSat 4 spacecraft [13], the solar array output was degrading at a much higher rate than expected which prompted a reanalysis of the environment and damage assessments. In this case, there were two payloads on-board the spacecraft to directly use on the analyses: 1) the Compact
Environment Anomaly Sensor (CEASE) [14] and 2) a solar cell experiment yielding full current-voltage (IV) data using similar solar cells and shielding conditions. Again, SCREAM was used successfully to analyze the CEASE data and predict the cell degradation, this time agreeing with the solar cell experiment data. Therefore, in this case, it was the environment which was much different than expected and two parallel payloads were used to confirm the higher rate of degradation experienced by the power panels.

It has become evident from these two examples that on-orbit displacement damage dosimetry is necessary to resolve anomalies and largely has been ignored in past space programs. There are several detector packages that can be used to generate displacement damage data such as CEASE [14] and CARMEN-2 [15]. Each of these packages are very powerful and can quantify several radiation hazards such as TID, spacecraft charging and SEE. However, each of these are very costly to integrate on a satellite and none directly yield displacement damage information, but are obtained through secondary means. For example, the CARMEN2 package contains an optically stimulated luminescent (OSL) detector from which the light output of a calibrated LED is used to quantify displacement damage.

The detectors and radiation effects section of NRL has recently been awarded a new-start program to investigate a detector that can easily be used to quantify displacement damage in real time onboard a spacecraft with low size, weight and power (SWaP). The Realtime Radiation Displacement Damage Dosimeter (R2D3) program is designed to accomplish this in 4 years and culminates in the production of a space qualified sensor for space use. There are several variations of the design which can accommodate varying degrees of sensitivity for both ground and space use. Different shielding schemes are being considered as well to encompass a wide energy range of the radiation environment. This work with be presented in a future publication.

The purpose of this paper is to demonstrate that when operating a GaAs diode in forward bias voltage mode, the measured dark current can be used as a metric 
for measuring the amount of absorbed displacement damage over a large proton energy range. In the present device, the forward biased dark current is dominated by generation-recombination in the junction. This simplifies the sensor's ground based calibration, improves accuracy, and also allows the device to be operated over a larger range of voltages thereby making this device an ideal candidate for a displacement damage dosimeter. Other advantages can be elucidated by different diode growth designs and gives some flexibility to the overall R2D3 system. The basic concepts of operation will be presented in this paper.

\section{EXPERIMENTAL DETAILS}

The samples used in this study were $\mathrm{p}^{+} \mathrm{n}$ GaAs photodiodes grown on GaAs substrates by molecular beam epitaxy. A detailed description of the photodiodes is given in [9]. The wafers were grown and processed at NRL. A sample consisted of three photodiodes $(2.4 \mathrm{~mm}$ diameter) and four solid diodes (1 $\mathrm{mm}$ in diameter). Current-voltage measurements were performed in the dark (DIV) on each sample both before and after irradiation. The DIV measurements were performed using a Keithley 2635A source measure unit using a four point probe technique both at NRL and at the radiation facilities when needed.

Two sets of irradiation campaigns were performed. The first set of irradiations were performed using 2 and $225 \mathrm{MeV}$ protons. The $2 \mathrm{MeV}$ proton irradiations were performed at the NRL Pelletron facility up to a maximum fluence of $10^{13} \mathrm{~h}^{+} / \mathrm{cm}^{2}$. The $225 \mathrm{MeV}$ proton irradiations were performed at the Francis H. Burr Proton Therapy Center to a maximum fluence of $3 \times 10^{14}$ $\mathrm{h}^{+} / \mathrm{cm}^{2}$. Different samples were irradiated at open circuit at several fluence levels and brought back to NRL for electrical characterization. Dosimetry was accomplished using Faraday cups in both facilities and all irradiations were performed at room temperature with low enough beam currents to avoid any significant increase in sample temperature. Typical flux values were $\sim 10^{9} \mathrm{~h}^{+} / \mathrm{cm}^{2} / \mathrm{sec}$. The 2 and $225 \mathrm{MeV}$ irradiations were performed in vacuum and air, respectively.

The second set of irradiations were performed to simulate the on-orbit response. The samples were exposed to $50 \mathrm{MeV}$ protons at the TRIUMF radiation facility located in Vancouver, BC. The samples were mounted in printed circuit boards and were wire bonded using 1 mil (25.4 micrometer) gold wires to enable in situ current measurements (Figure 1). Each device was connected to enable four point probe measurements. Two irradiation experiments were performed. For the first one, full DIV data were taken prior to and following incremental fluences to develop full characteristic degradation data (calibration curve). For the second one, full DIV data were taken in situ while the beam was on (during proton exposure). These the

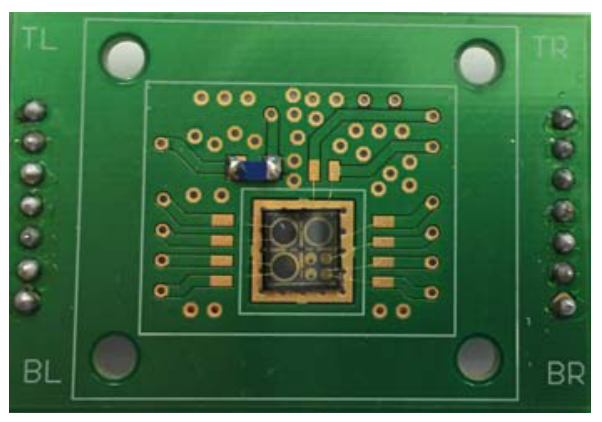

Figure 1. Image showing a sample (5 devices) mounted and wire bonded in a printed circuit board for in situ characterization.

measured current values were then used to predict corresponding fluence values (via DDD) using the calibration data. The typical beam flux at TRIUMF was $10^{9} \mathrm{~h}^{+} / \mathrm{cm}^{2} / \mathrm{sec}$ but was reduced to $10^{8} \mathrm{~h}^{+} / \mathrm{cm}^{2} / \mathrm{sec}$ during in situ characterization to eliminate proton-induced beam current effects. These irradiations were also performed at room temperature and in air. Dosimetry was accomplished using an ion chamber.

\section{EXPERIMENTAL RESULTS}

3.1 Characteristic Degradation Curve Development

The response of the GaAs diode dark current as a function of forward bias voltage both prior to and after 2 $\mathrm{MeV}$ and $225 \mathrm{MeV}$ proton irradiation with proton fluences up to $2 \times 10^{14} \mathrm{~cm}^{-2}$ are shown in Figure 2 and Figure 3, respectively. The dashed line represents the pre-radiation DIV response while the solid lines correspond to DIV response after proton exposure with increasing fluence levels. In all cases, the dark current increases with increasing proton fluence over the entire voltage range. Figure 4 shows the same data in Figure 2 where the measured dark current values at fixed voltage bias points $0.2 \mathrm{~V}-0.8 \mathrm{~V}$ ) are plotted as a function of 2 $\mathrm{MeV}$ proton fluence. There is a noted linear dependence in current as a function of the fluence for all bias voltage points shown. The 2 and $225 \mathrm{MeV}$ proton dark current data are combined and plotted as a function of DDD in Figure 5 using total NIEL data for GaAs [2]. The NIEL values for 2 and $225 \mathrm{MeV}$ protons on GaAs is $2.89 \times 10^{-2}$ and $3.21 \times 10^{-3} \mathrm{MeV} \mathrm{cm} 2 / \mathrm{g}$. The linearity of the data with DDD is seen to extend $\sim 1$ order of magnitude by adding the $225 \mathrm{MeV}$ data to the plot.

The data in Figure 5 can now be classified as characteristic degradation data, or calibration data, representing the dark current increase for these particular GaAs p-n junction diodes. The effects of proton irradiation on this device can be determined using the calibration data in conjunction with the proton NIEL. The fact that these data are linear over a large proton energy range and a wide voltage bias range gives us good confidence that this device structure is an excellent candidate for a displacement damage 


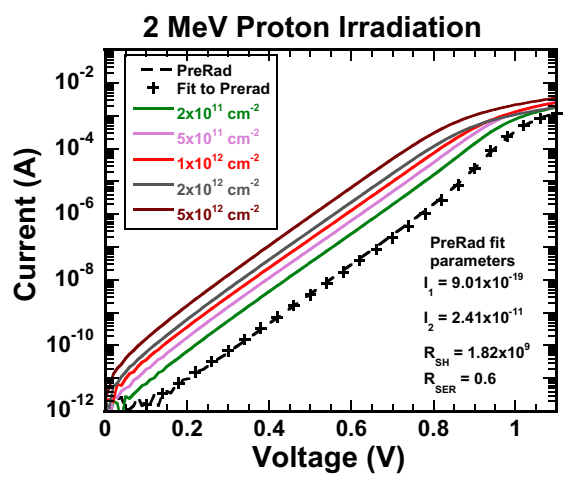

Figure 2. Dark current-voltage response of a $\mathrm{p}^{+} \mathrm{n}$ GaAs photodiode following $2 \mathrm{MeV}$ proton irradiation up to a fluence level of $5 \times 10^{12} \mathrm{~cm}^{-2}$. The pre-irradiation curve is represented by the dashed line, and the + symbols represent a fit using equation 1. The other lines correspond to increasing fluence levels and are not fits. Data is plotted as lines to clearly illustrate trends. The data resolution is $10 \mathrm{mV}$.

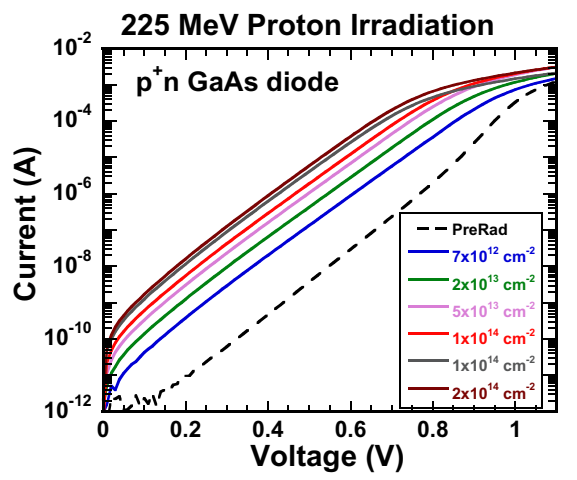

Figure 3. Dark current-voltage data of a $\mathrm{p}^{+} \mathrm{n}$ GaAs photodiode following $225 \mathrm{MeV}$ proton irradiation up to a fluence level of $2 \times 10^{14} \mathrm{~cm}^{-2}$. The pre-irradiation curve is represented by the dashed line. The other lines correspond to increasing fluence levels and are not fits. Data is plotted as lines to clearly illustrate trends. The data resolution is $10 \mathrm{mV}$.

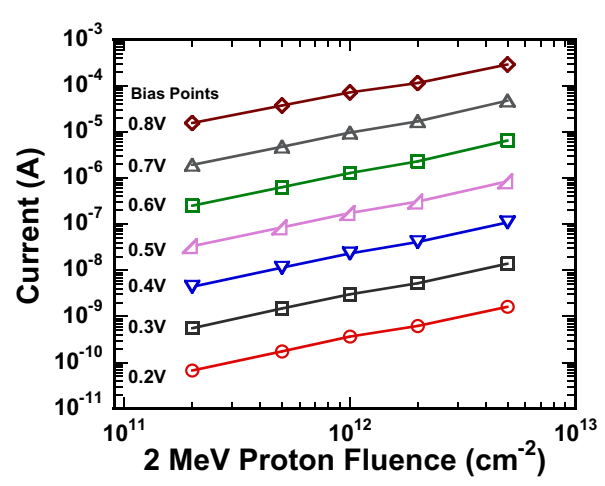

Figure 4. Measured current values at fixed voltages (0.2-0.8 $\mathrm{V})$ as a function of $2 \mathrm{MeV}$ proton fluence for $\mathrm{p}^{+} \mathrm{n}$ GaAs photodiodes. These data were extracted from Figure 2.

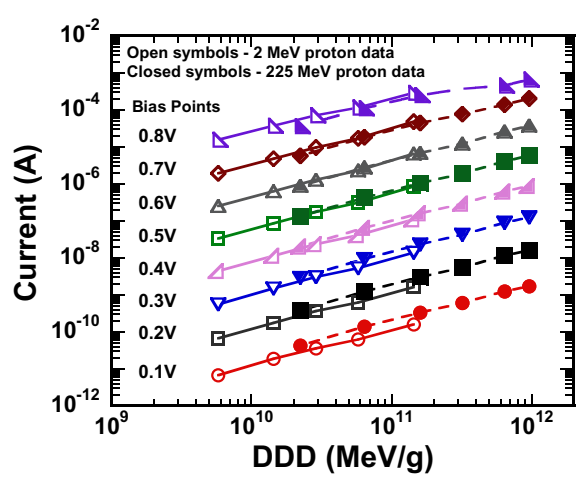

Figure 5. Measured current values at fixed voltages as a function of displacement damage dose for $\mathrm{p}^{+} \mathrm{n}$ GaAs photodiodes. Data were extracted from Figure 2 and Figure 3. The open and closed symbols correspond to the $2 \mathrm{MeV}$ and $225 \mathrm{MeV}$ proton data, respectively. Each curve corresponds to a different bias voltage.

dosimeter for either monoenergetic or spectral proton energies. The data at low and high bias values do show some effects of nonlinearity and can be explained by the presence of shunt and series resistance effects, respectively. Nonetheless, the acceptable voltage range of linearity is $\sim 0.4-0.7 \mathrm{~V}$ which gives an excellent trade space in absolute current measurement capabilities. That is, different bias values can be applied to this structure to bring the current into a desired measurement range in the final circuit of the R2D3. A full voltage sweep would provide a lot of data to work with in practice.

\subsection{Simulation of On-Orbit Performance}

To simulate the on-orbit response and check the general feasibility that this system will work in space, additional proton irradiations were performed on GaAs devices. The devices were packaged (see Figure 1) so the diode dark currents could be measured in situ during proton exposure. The irradiations were performed using 50 $\mathrm{MeV}$ protons at TRIUMF. Two sets of radiation experiments were performed in this effort. The first set of experiments set out to define the characteristic dark current degradation data (similar to the data in Figure 5) of which the results are shown in Figure 6. The fluence values were converted to DDD using a $50 \mathrm{MeV}$ proton NIEL value of $4.11 \times 10^{-3} \mathrm{MeV} \mathrm{cm}^{2} / \mathrm{g}$.

The current data shown in Figure 6 has three functional dependencies (regions) with displacement damage dose in contrast to the data of Figure 5 where only a linear dependence is observed. For the $50 \mathrm{MeV}$ proton data set, the radiation experiments started at much lower fluence values in order to find the onset of measurable dark current increase. In region 1, there is a minimal change in dark current (mechanism unknown) until the DDD reaches $\sim 7 \times 10^{7} \mathrm{MeV} / \mathrm{g}$. Thereafter, there 


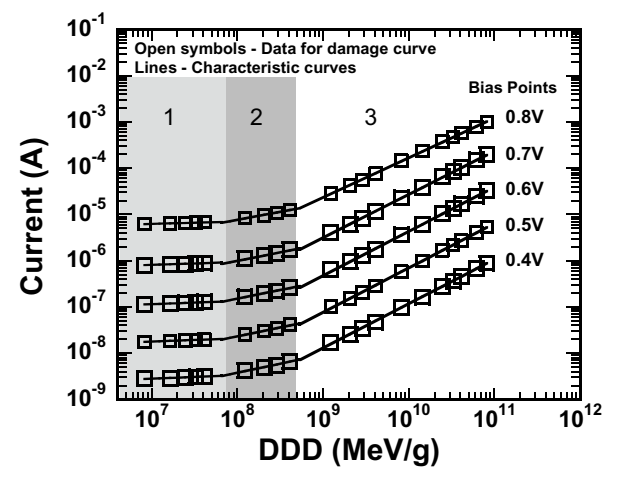

Figure 6. Dark current as a function of displacement damage dose for $50 \mathrm{MeV}$ proton irradiations at Triumf. The data points correspond to the characteristic damage curves at each bias voltage and lines are coplotted at various slopes to guide the eye.

is an increase in slope as defined by region 2 until $\sim 6 \times 10^{8} \mathrm{MeV} / \mathrm{g}$. Region 3 is then defined showing the linear behavior with the dark current and DDD. Region 3 is consistent with the dark current being dominated by recombination processes also presented in Figure 5. The slope of the curve in region 2 is consistent with the dark current being dominated by diffusion processes. This was previously not observed in earlier datasets because experiments were not carried out at these lower DDD values. The lines in Figure 6 for region 2 and 3 are the characteristic damage curves at each bias point determined from fits using equations (4) and (5), which are presented below.

The second set of experiments taken at TRIUMF aimed to test how well the measured dark current can be used to predict the particle fluence (DDD) using the characteristic degradation data generated from the first set of experiments. The beam fluence at the facility is measured in terms of monitor counts. Full dark current voltage sweeps were measured with the $50 \mathrm{MeV}$ proton beam on in situ at random increasing monitor counts. The monitor counts were converted to a known fluence level. These results for several discrete forward bias voltages are shown in Figure 7 where the lines represent the characteristic degradation data (first experiment) and the data points represent the in situ measured dark current. The agreement is excellent over a large DDD and voltage bias range. An estimate of errors can be obtained through these experiments. The predicted DDD as a function of actual DDD is plotted in Figure 8. The different symbol types correspond to current values measured at different forward bias voltages. The predicted DDD was calculated using the characteristic degradation data curves (first experiment) and the dark current that was measured in situ (second experiment). The solid line represents the actual fluence values. Data above and below this line correspond to an overestimate and underestimate, respectively.

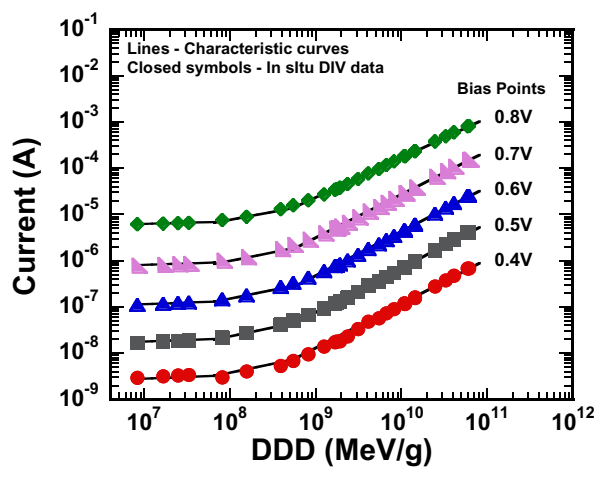

Figure 7. Dark current as a function of DDD. The lines correspond to the characteristic damage curves at each bias voltage and the symbols represent the dark current data measured in situ during $50 \mathrm{MeV}$ proton exposures.

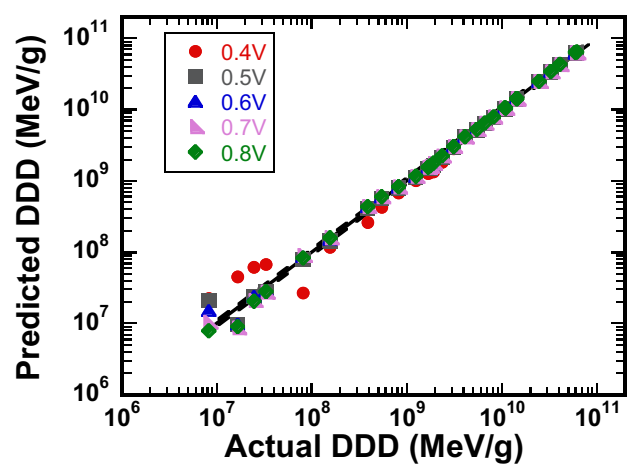

Figure 8. Predicted DDD as a function of actual DDD. The solid line represents the actual DDD. Data points below the line correspond to underestimate of the DDD and points above are overestimates. The two dashed lines are representative of $\pm 10 \%$.

To quantitatively access the uncertainty in using the dark current to predict the DDD on-orbit, a plot showing the percent error is shown in Figure 9. The horizontal dashed lines represent $\pm 10 \%$ error. For the most part, the data are bound by these limits with the exception of $\mathrm{DDD}$ values below $\sim 3 \times 10^{7} \mathrm{MeV} / \mathrm{g}$ and the $0.4 \mathrm{~V}$ dataset. The percent error actually decreases for increasing DDD values.

The reason for the large deviations at low DDD values is evident from Figure 6. For DDD values below $3 \times 10^{7} \mathrm{MeV} / \mathrm{g}$, a small change in measured current leads to a large uncertainty in the predicted DDD value because of the very steep slope of the characteristic degradation data evident when plotted as DDD vs dark current. When the current degradation mechanism changes from being diffusion current dominated to recombination current dominated, there is a decrease in the slope of the characteristic damage curve with a corresponding reduction in uncertainty in the predicted DDD. Although there is a large percent error for DDD values below $3 \times 10^{7} \mathrm{MeV} / \mathrm{g}$, this would not be so 


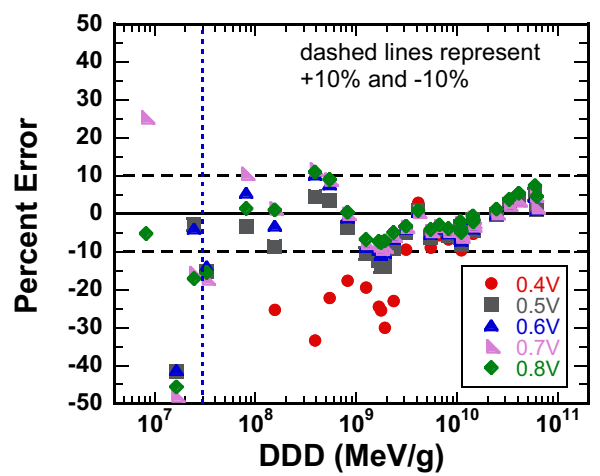

Figure 9. Calculated percent error between the predicted and actual DDD. The horizontal dashed lines represent $\pm 10 \%$. The data points correspond to data measured at fixed voltage values.

important for most space missions. For example, consider the GPS orbit. A dose value of $\sim 3 \times 10^{7} \mathrm{MeV} / \mathrm{g}$ (indicated by vertical line in Figure 9), which is $\sim$ the sensitivity limit of the current sensor design, would be reached in $\sim 0.5$ days in orbit if this device had $1 \mathrm{mil}$ of aluminum shielding. This dose value would be reached much sooner for device with no shielding. Therefore, this sensor can be used for real-time DDD monitoring. Since this dose value is achieved fairly early in most missions, the large uncertainties observed at the low dose values are not important for space applications. Also, the same DDD value can be predicted at different forward bias voltages so the accuracy could be improved by averaging these DDD values.

\section{DISCUSSION}

The results presented in Section 3 can be qualitatively explained using conventional $\mathrm{p}-\mathrm{n}$ junction theory. The forward-biased current in the dark of a junction is given by [16]:

$$
\begin{aligned}
I(V) & =I_{1}\left[e\left(\frac{V-I R_{S E R}}{k_{b} T}\right)-1\right]+ \\
& I_{2}\left[e\left(\frac{V-I R_{S E R}}{2 k_{b} T}\right)-1\right]+\frac{V-I R_{S E R}}{R_{S H}}
\end{aligned}
$$

where $I_{1}$ is the diffusion current constant in the quasineutral regions of the device, $I_{2}$ is the recombinationgeneration current constant in the space charge region (SCR), $R_{S E R}$ is the series resistance, $R_{S H}$ is the shunt resistance, $k_{B}$ is the Boltzmann constant and $T$ is the temperature. The prefactors $I_{1}$ and $I_{2}$ can be expressed as

$$
\begin{gathered}
I_{1}=\frac{q n_{i}^{2} \sqrt{D_{n}}}{N_{A} \sqrt{\tau_{n}}}+\frac{q n_{i}^{2} \sqrt{D_{p}}}{N_{D} \sqrt{\tau_{p}}} \\
I_{2}=\frac{n_{i} k T}{\sqrt{\tau_{h} \tau_{n}}} \frac{W_{0}}{\sqrt{V_{b}}} \frac{\pi}{2}
\end{gathered}
$$

$D_{n, p}$ are the electron and hole diffusion coefficients, respectively, $n_{i}$ is the intrinsic carrier concentration, $\tau_{n, p}$ are the minority carrier life time in the $\mathrm{n}$ - and $\mathrm{p}$-regions, respectively, $N_{A, D}$ are the acceptor and donor doping concentrations on each side of the junction, $V_{b}$ is the built-in voltage and $W_{0}$ is the width of the SCR with no applied bias. The diffusion and recombination current constants can be simplified and related to particle fluence with the following substitutions: $L^{2}=D \tau$ and for $\tau=\left(N_{T} \sigma v_{t h}\right)^{-1}$ with the defect concentration given by $N_{T}=k \varphi$ (assuming linear trap introduction rates), (2) and (3) can be re-written as:

$$
\begin{aligned}
& I_{1}=I_{1}{ }^{*} \varphi^{1 / 2} \\
& I_{2}=I_{2}{ }^{*} \varphi
\end{aligned}
$$

where $I_{1}{ }^{*}$ and $I_{2}{ }^{*}$ are defined in [16]. Equations (4) and (5) indicates that the diffusion dark current constant has a square root dependence with particle fluence while the recombination dark current constant is linear relationship with fluence (or displacement damage dose when fluence is converted to DDD using NIEL).

Each dark current dataset was fit to equation 1 using a nonlinear least squares fitting routine to determine the fitting parameters $I_{1}$ and $I_{2}, R_{S H}$, and $R_{S E R}$. An example of such a fit is shown in Figure 2 represented by the + symbols. The values for the fitting constants for the pre-radiation data are given in the figure. The values for the fitting constants to the preradiation data are shown in the figure. Depending on the device technology, structure, and measurement voltage range, the forward bias dark current can be characterized by 4 regions: 1) diffusion, 2) recombination, 3) shunt resistance and 4) series resistance. The dark current due to recombination in the depletion region usually dominates at low voltage bias and the dark current associated with diffusion in the quasi-neutral region dominates at higher voltage bias regions. The magnitude where each mechanism contributes to the forward bias current depends on varied parameters such as the material type, doping and layer thicknesses. For the GaAs diodes in the present work, these regions are illustrated in the pre-radiation data. For $\mathrm{V}<0.05 \mathrm{~V}$, shunt resistance dominates the current. From $0.05<\mathrm{V}<0.75$ the recombination current $\left(n_{2}=2\right)$ dominates. Thereafter, there is an increase in the slope when the diffusion current $\left(n_{l}=1\right)$ begins to dominate, and eventually series resistance dominates the current flow causing a "roll-over" or flattening effect which is more evident in radiation data than the preradiation data set for this data set. However, after irradiation, the slope is fairly constant over a large bias range $(0.1 \mathrm{~V}$ to $0.8 \mathrm{~V})$ indicating that the current is dominated by one of the mechanisms discussed above.

Prior to irradiation, the diffusion current constant $I_{1}$ was already $\sim 8$ orders of magnitude smaller than the recombination component $\mathrm{I}_{2}$. After irradiation, the recombination current continued to dominate the forward bias current. The dark current in these GaAs 


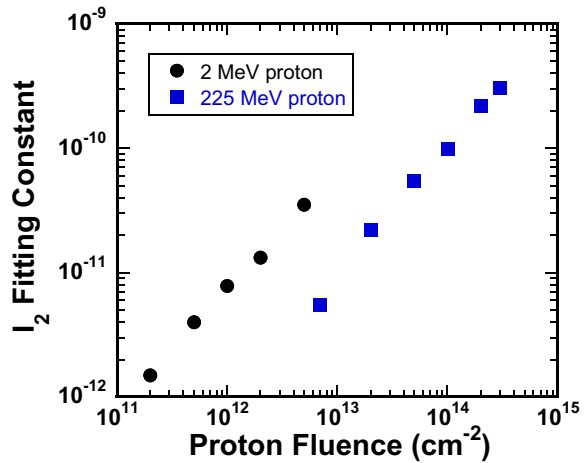

Figure 10. $I_{2}$ fitting term as a function of proton fluence for the $2 \mathrm{MeV}$ and $225 \mathrm{MeV}$ proton datasets. Each dataset was fit using equation 1 to extract the I 2 fitting term.

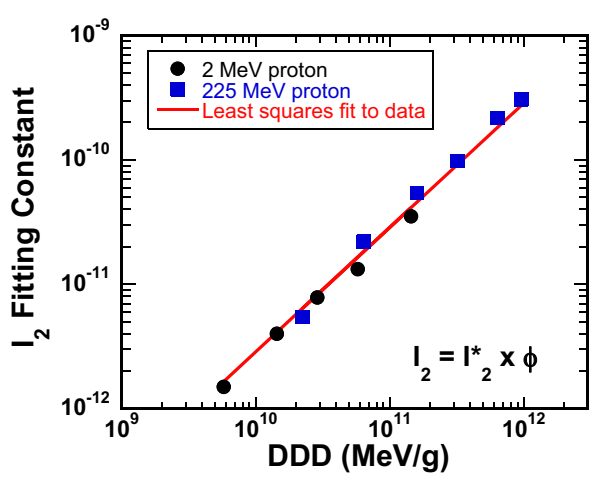

Figure 11. $I_{2}$ fitting term as a function of displacement damage dose. The data collapse and can be represented by a single characteristic curve defined by equation 5 (fluence in this equation must be converted to displacement damage dose).

diodes over this fluence range is thereby dominated by Shockley-Read-Hall recombination (SRH) in the SCR. The fitting results for $\mathrm{I}_{2}$ as a function of proton fluence is shown in Figure 10 for both the $2 \mathrm{MeV}$ and $225 \mathrm{MeV}$ proton data. It is clear that the DIV response depends on the both the proton energy and fluence. These same data have been re-plotted in Figure 11 as a function of DDD by multiplying the proton fluence by the corresponding NIEL value for protons in GaAs $\left(2.89 \times 10^{-2}\right.$ and $3.21 \times 10^{-3} \mathrm{MeVcm}^{2} / \mathrm{g}$ for 2 and $225 \mathrm{MeV}$ protons, respectively). When plotted as a function of DDD, the data collapse and can be represented by a single characteristic curve (solid line in Figure 11). The rate at which defects are introduced and their effect on the forward bias dark current is linearly proportional to the total NIEL.

The basic mechanisms and dependence of the diffusion and recombination current constants with particle fluence can be qualitatively explained by fitting the data in Figure 11 to equations. (4) or (5). The data in were fit using equation. (5) and the slope was determined to be 1.013 , which confirms the dark current of this diode over this DDD range is dominated by recombination in the depletion region. However, the $50 \mathrm{MeV}$ proton data set in Figure 6 shows that the current increase is dominated be either diffusion (region 2) or recombination (region 3) processes depending on the DDD value.

\section{R2D3 DEVELOPMENT}

The in situ electrical characterization has given confidence that the device can be used in a space environment to predict DDD. There does not appear to be any current injection annealing and GaAs devices are also well-known to be insensitive to thermal annealing until $\sim 200^{\circ} \mathrm{C}$. The R2D3 development of the prototype is currently underway. It is desired to have data collection, storage and telemetry as well as temperature measurement contained in the R2D3 package. For the measurement electronics, different levels of processing complexity in the implementation are being explored. At the high end of complexity, we are looking at a radiation-hardened version of the 8051 microcontroller. This is the same microcontroller used on the Microelectronics and Photonics Test Bed (MPTB) experiment payload. An alternative approach to this would be a rad-hard Field Programmable Gate Array (FPGA) that implements a simple state machine to make the necessary analog measurements and send the data to the host spacecraft. In both cases, a rad-hard highresolution analog-to-digital converter will make the necessary measurements.

\section{CONCLUSIONS}

A characteristic degradation curve of the dark current in irradiated GaAs p-n junction diodes was obtained as a function of DDD following proton irradiations from 2 to $225 \mathrm{MeV}$. The linearity of the characteristic damage curve gives credence to a novel DD dosimeter for space applications. Furthermore, the wide voltage bias range over which the linearity is obtained simplifies the on-orbit measurement requirements. Additional proton testing at $50 \mathrm{MeV}$ was performed to simulate measurements in space and yielded error estimates within $10 \%$ of expected values. The R2D3 system will contain the GaAs device with the associated measurement electronics for data collection, storage and telemetry and offers a low SWaP and low cost option for DD dosimetry for space and ground applications. A full voltage sweep in orbit will yield a large amount of data to improve error estimates. Future research will involve electron testing and temperature effects.

\section{ACKNOWLEDGEMENTS}

The authors would like to sincerely thank TRIUMF 
radiation facility for the proton beam time in order to carry out the $50 \mathrm{MeV}$ proton radiation experiments.

\section{REFERENCES}

[1] G. P. Summers, E. A. Burke, P. Shapiro, S. R. Messenger, and R. J. Walters, "Damage correlations in semiconductors exposed to gamma, electron and proton radiations," IEEE Trans. Nucl. Sci., vol. 40, no. 6, pp. 1372-1379, 1993.

[2] J. Insoo, M. A. Xapsos, S. R. Messenger, E. A. Burke, R. J. Walters, G. P. Summers, and T. Jordan, "Proton nonionizing energy loss (NIEL) for device applications," IEEE Trans. Nucl. Sci., vol. 50, no. 6, pp. 1924-1928, 2003.

[3] J. Insoo, K. Wousik, and R. Evans, "Electron Nonionizing Energy Loss for Device Applications," IEEE Trans. Nucl. Sci., vol. 56, no. 6 , pp. 3229-3235, 2009.

[4] G. P. Summers, E. A. Burke, and M. A. Xapsos, "Displacement damage analogs to ionizing radiation effects," Radiation Measurements, vol. 24, no. 1, pp. 1-8, 1995.

[5] C. Inguimbert, and S. Messenger, "Equivalent Displacement Damage Dose for On-Orbit Space Applications," IEEE Trans. Nucl. Sci., vol. 59, no. 6, pp. 3117-3125, 2012.

[6] S. R. Messenger, G. P. Summers, E. A. Burke, R. J. Walters, and M. A. Xapsos, "Modeling solar cell degradation in space: A comparison of the NRL displacement damage dose and the JPL equivalent fluence approaches," Progress in Photovoltaics: Research and Applications, vol. 9, no. 2, pp. 103-121, 2001.

[7] J. H. Warner, S. R. Messenger, R. J. Walters, and G. P. Summers, "Displacement damage correlation of proton and silicon ion radiation in GaAs," IEEE Trans. Nucl. Sci., vol. 52, no. 6, pp. 2678-2682, 2005.

[8] J. H. Warner, S. R. Messenger, R. J. Walters, G. P. Summers, J. R. Lorentzen, D. M. Wilt, and M. A. Smith, "Correlation of Electron Radiation Induced-Damage in GaAs Solar Cells," IEEE Trans. Nucl. Sci., vol. 53, no. 4, pp. 1988-1994, 2006.

[9] J. H. Warner, R. J. Walters, S. R. Messenger, G. P. Summers, S. M. Khanna, D. Estan, L. S. Erhardt, and A. Houdayer, "High-energy proton irradiation effects in GaAs devices," IEEE Trans. Nucl. Sci., vol. 51, no. 5, pp. 2887-2895, 2004.

[10] S. R. Messenger, E. M. Jackson, J. H. Warner, and R. J. Walters, "Scream: A new code for solar cell degradation prediction using the displacement damage dose approach," IEEE
2010 35th Photovoltaic Specialists Conference (PVSC). pp. 001106-001111, 20-25 June 2010.

[11] S. R. Messenger, E. A. Jackson, J. H. Warner, and R. J. Walters, "Advancements to SCREAM: Multiple spectrum input and ShielDDDose options," IEEE 2012 38th Photovoltaic Specialists Conference (PVSC). pp. 003281-003286, 3-8 June 2012.

[12] S. R. Messenger, E. M. Jackson, J. H. Warner, R. J. Walters, T. E. Cayton, C. Yue, R. W. Friedel, R. M. Kippen, and B. Reed, "Correlation of Telemetered Solar Array Data With Particle Detector Data On GPS Spacecraft," IEEE Trans. Nucl. Sci., vol. 58, no. 6, pp. 3118-3125, 2011.

[13] P. P. Jenkins, D. C. Bentz, J. Barnds, C. R. Binz, M. S.R., W. J.H., M. J. Krasowski, N. F. Prokop, D. C. Spina, M. O’Neill, M. Eskenazi, H. W. Brandhorst, E. Downard, and K. C. Crist, "TACSAT-4 Solar Cell Experiment: Two Years in Orbit," Proceedings of 10th European Space Power Conference, 13-17 April 2014

[14] B. K. Dichter, J. O. McGarity, M. R. Oberhardt, V. T. Jordanov, D. J. Sperry, A. C. Huber, J. A. Pantazis, E. G. Mullen, G. Ginet, and M. S. Gussenhoven, "Compact environmental anomaly sensor (CEASE): a novel spacecraft instrument for in situ measurements of environmental conditions," IEEE Trans. Nucl. Sci., vol. 45, no. 6, pp. 2758-2764, 1998.

[15] C. Deneau, J. R. Vaille, L. Dusseau, J. Mekki, P. Garcia, F. Bezerra, E. Lorfevre, and R. Ecoffet, "First In-Flight Data Analysis of Displacement Damage on the OSL Sensor OnBoard CARMEN-2," IEEE Trans. Nucl. Sci., vol. 58, no. 3, pp. 939-944, 2011.

[16] J. C. Bourgoin, and M. Zazoui, "Irradiationinduced degradation in solar cell: characterization of recombination centres," Semiconductor Science and Technology, vol. 17, no. 5, pp. 453, 2002. 